\title{
Using affinity propagation for identifying subspecies among clonal organisms: lessons from M. tuberculosis
}

\author{
Claudio Borile ${ }^{1,2}$, Mathieu Labarre ${ }^{1}$, Silvio Franz ${ }^{1}$, Christophe Sola ${ }^{3,4}$ and Guislaine Refrégier ${ }^{3^{*}}$
}

\begin{abstract}
Background: Classification and naming is a key step in the analysis, understanding and adequate management of living organisms. However, where to set limits between groups can be puzzling especially in clonal organisms. Within the Mycobacterium tuberculosis complex (MTC), the etiological agent of tuberculosis (TB), experts have first identified several groups according to their pattern at repetitive sequences, especially at the CRISPR locus (spoligotyping), and to their epidemiological relevance. Most groups such as "Beijing" found good support when tested with other loci. However, other groups such as T family and T1 subfamily (belonging to the "Euro-American" lineage) correspond to non-monophyletic groups and still need to be refined. Here, we propose to use a method called Affinity Propagation that has been successfully used in image categorization to identify relevant patterns at the CRISPR locus in MTC.

Results: To adequately infer the relative divergence time between strains, we used a distance method inspired by the recent evolutionary model by Reyes et al. We first confirm that this method performs better than the Jaccard index commonly used to compare spoligotype patterns. Second, we document the support of each spoligotype family among the previous classification using affinity propagation on the international spoligotyping database SpolDB4. This allowed us to propose a consensus assignation for all SpolDB4 spoligotypes. Third, we propose new signatures to subclassify the T family.

Conclusion: Altogether, this study shows how the new clustering algorithm Affinity Propagation can help building or refining clonal organims classifications. It also describes well-supported families and subfamilies among $M$. tuberculosis complex, especially inside the modern "Euro-American" lineage.
\end{abstract}

Keywords: asexual organisms, species delineation, epidemiology, DR locus, Clustered Regularly Interspaced Short Palindromic Repeats (CRISPR)

\section{Background}

The advent of powerful genotyping methods, either by global sequencing or by high-throughput analysis of variation at specific loci (mini- or micro-satellites [1]; CRISPR (Clustered Regularly Interspaced Short Palindromic Repeats) loci [2,3]; SNPs [4]), provides masses of genetic data that need to be compared and clustered. Most widely used comparison methods are phylogenetic methods i.e. hierarchical clustering, building tree-like

\footnotetext{
* Correspondence: guislaine.refregier@u-psud.fr

${ }^{3}$ IGM, CNRS and Univ. Paris-Sud, UMR8621, Bat. 400, F-91405 Orsay cedex, France

Full list of author information is available at the end of the article
}

structures to display the diversity. These methods consider that each individual forms a cluster and repeatedly merge the most similar clusters based on pairwise distances (Phenetics such as Neighbour-Joining), or try to infer the tree that most fits the data (Cladistics such as Maximum Likelihood, Bayesian analysis) using an appropriate evolutionary model of the compared characters. This provides a continuous pattern of how divergent organisms are. Other comparison methods consist in finding relevant clusters, a process referred to as partitioning. A method made popular by the software Structure [5], and referred to as model-based clustering, consists in using Bayesian methods to assign individuals
C Biomed Central

() 2011 Borile et al; licensee BioMed Central Ltd. This is an Open Access article distributed under the terms of the Creative Commons Attribution License (http://creativecommons.org/licenses/by/2.0), which permits unrestricted use, distribution, and reproduction in any medium, provided the original work is properly cited. 
in a pre-determined number of populations. The assumption underlying this software is that the population conforms Hardy-Weinberg hypotheses i.e. refers to organisms reproducing sexually, with random pairing inside the population. This assumption is theoretically very problematic for clonal organisms, although practice has shown that it can provide meaningful results [6], partly because some parameters can be set to mimic poor mixture inside populations. Other methods have been developed outside biology, for instance to categorize images $[7,8]$. They use similarity to group data in spherical clusters well represented by their centroid (also called representative or exemplars), and have already been tentatively used to classify human genetic data [9]. This method awaits further experimental validation on large datasets.

Clustering methods can be applied to different types of loci, ranging from repetitive sequences such as insertion sequences, micro-, mini-satellites or the CRISPR loci to single nucleotide polymorphisms (SNPs), provided an appropriate method is available to calculate the distance between individuals. Such methods usually rely on a model of the mutation process. Which loci should be targeted depends on the mean divergence time between individuals, as repetitive sequences mutate faster than SNP loci. Several mutation models have been developed for DNA sequence with point mutations [10]. For repetitive sequences (micro- and mini -satellites), categorical distance or the Stepwise Mutation Model (SMM [11]) are mostly used.

CRISPR loci (Clustered Regularly Interspaced Short Palindromic Repeats) form a new family of repetitive sequences [12,13]. They consist in the repetition of 24 to $47 \mathrm{bp}$ sequences called Direct Repeats (DR) separated by unique sequences called spacers (from 26 to $72 \mathrm{bp}$ ). The constitutive unit is therefore the combination of one DR and one spacer, and presently described CRISPR loci have from 2 to 249 units [13]. These repetitions are surrounded by protein-encoding sequences called cas genes (derived from CRISPR-associated genes). The whole locus confers resistance to bacteriophages and plasmids in Streptococcus thermophilus [14,15] and in Escherichia coli when overexpressed [16]. Resistance to the corresponding organisms is under investigation in species where spacers are homologous to foreign DNA [17]. They exhibit a quite high mutation rate so that they have proven useful for epidemiological studies. Describing the presence or absence of 43 spacers of $M$. tuberculosis CRISPR locus has become a routine technique in any tuberculosis reference center and is referred to as spoligotyping for spacer oligonucleotide typing [18]. Pairwise comparisons of binary profiles can provide a distance matrix that has been used to infer phylogenetic relationships. The most common approach to infer relationships using spoligotype patterns uses the Jaccard index (same principle as Hamming distance or Dice coefficient) as distance [19], counting the proportion of spacers that are present in both profiles. The distance matrix, either made of pure spoligotyping data or combining them with minisatellite data, is usually processed using UPGMA or NJ algorithm to build a dendrogram or a phylogenetic tree [20]. A more elaborate approach using the Zipf distribution and the evolutionary dynamics of CRISPR loci has proven more relevant to infer phylogenetic relationships for the M. tuberculosis complex [21] but is not implemented in a user-friendly software yet and does not propose assignations for all currently described spoligotype patterns.

The worldwide database of spoligotyping in $M$. tuberculosis complex is called SpolDB (the latest public version being SpolDB4), and has helped identifying recurrent signatures in CRISPR profiles [22-24]. These signatures, mainly based on the absence of adjacent spacers, led to the naming of large clonal families, the monophyly of which has been confirmed through other markers such as minisatellites (referred to as MIRUVNTR for Mycobacterial-Interspersed-Repetitive-UnitsVariable Number of Tandem Repeats), Region of Deletions (RDs) and SNPs [6,25,26]. Main acknowledged families are EAI for East-African-Indian (later referred to as "Indo-Oceanic" by Gagneux et al.), M. africanum 1 and 2 (later "West-african 2" and "West-African 1"), animal strains ( $M$. bovis, $M$. caprae, $M$. pinnipedii, $M$. microtii), CAS for "Central-Asia" (later "East-AfricanIndian"), Beijing, X, Haarlem, LAM for "Latino-American and Mediterranean", $T$ and MANU (also designated as $\mathrm{T}$ ancestor) [23,27]. Monophyly of each of the LAM, $\mathrm{T}$ and Haarlem families has been partly invalidated. However, the larger lineage to which they belong and that is characterized by the 33-36 spacers deletion at the CRISPR profile (merging T, LAM, X, Haarlem families and $S$ subfamily) has been confirmed and designated as the "Euro-American" lineage [27]. It corresponds to the Principal Genetic Groups (PGG) 2 and 3 as defined by Sreevatsan et al. [28]. Altogether deletions in spoligotype patterns have proven to contain phylogenetic information and allow most strains be assigned to the families described above. Assignations performed by experts are reported in SpolDB4 database, patterns carrying no or contradictory signatures been labeled as "U" for "Unknown or Unclassified". To circumvent the dependence on experts' analysis, the Bennett's laboratory proposed automatized classification of spoligotype patterns using Bayesian algorithms and a distance method taking into account the deletion process by which spoligotype patterns evolve. They provide an on-line tool called Spotclust [29] to assign each spoligotype to a family, either one described in SpolDB3 
[30] or one of the 6 large families proposed by Gagneux and coworkers [31].

Here we wanted to take advantage of a recently developed algorithm, Affinity Propagation, to confirm and extend these methods. This algorithm identifies references for every data point so that data are grouped and centered on these references while a specific cost function is minimized. The cost of adding a new reference point, assigned by the user, determines the final number of clusters. Prior to the use of this algorithm, we tested different distances to calculate pairwise distances between spoligotype patterns. We took advantage of previously identified references and expert assignation to rank these distances, some of which are derived from previously proposed evolutionary models [21,31]. The distance that best identified the appropriate reference for each spoligotype pattern was implemented in the Affinity Propagation algorithm to identify relevant subfamilies among $M$. tuberculosis complex (MTC). These families partly correlated with previously identified subfamilies.

Altogether, this approach allowed us to assess the robustness of previously identified sublineages among MTC, to identify new relevant sublineages and to provide re-assignations of the spoligotype patterns described in SpolDB4. These re-assignations interestingly matched those of studies using VNTR and/or SNP data.

\section{Results}

Comparison of classifications based on new distances or on Jaccard index to expert classification of SpolDB4

Clustering of CRISPR patterns (spoligotypes) of $M$. tuberculosis complex is commonly done using the Jaccard index as distance [32]. This index counts the shared spacers without taking into account their spatial organization. However, it has been shown that adjacent spacers have a higher probability to be simultaneously deleted [21], and this feature has been used by experts to define $M$. tuberculosis complex families and subfamilies [22,23] in the international database SpolDB [33]. We wanted here to identify a distance conducing to the best concordance of spoligotype assignations at the family level, as available in SpolDB4 database [23]. We retained the ten widely acknowledged families: $M$. africanum, Animal strains (grouping M. bovis, M. pinnipedii, $M$ microtii, and $M$ caprae), Beijing (herein also referred to as Beij), CAS, EAI, Haarlem (also referred to as $\mathrm{H}$ ), LAM, MANU, T and X [25]. Each is characterized by a different spoligotype signature and thus a different reference profile $[22,33]$ (Table 1 ). In addition to Jaccard index, we set up three methods to compute the distance between pairs of patterns: "Domain Walls" measuring the proportion of shared limits of blocks of
Table 1 References of the ten best acknowledged $M$. tuberculosis complex families

\begin{tabular}{llll}
\hline SIT & \multicolumn{2}{l}{ SpolDB4 classification } & Reference Spoligotype pattern \\
\cline { 2 - 3 } & family & subfamily & \\
\hline 1 & BEIJ & BEIJ & \\
26 & CAS & CAS1 & \\
42 & LAM & LAM9 & \\
50 & H & H3 & \\
53 & T & T1 & \\
100 & MANU & MANU1 & \\
119 & X & X1 & \\
236 & EAI & EAI5 & \\
181 & AFRI & AFRI1 & \\
482 & animal & BOV1 &
\end{tabular}

BEIJ = Beijing also referred to as East Asia; CAS = Central Asia also referred to as East Africa and India; LAM = Latino-American and Mediterranean; $\mathrm{H}=$ Haarlem; EAI = East African Indian.

spacers in the CRISPR locus, "Blocks" measuring the proportion of shared blocks of spacers, and "Deletions" measuring the proportion of shared blocks of deleted spacers (see Methods and Figure 1). We implemented these four methods to compute the distances of each spoligotype of SpolDB4 database [33] to the reference profiles of the ten families (see Table 1). For each method, depending on the reference to which it was most similar, each spoligotype was assigned to one of

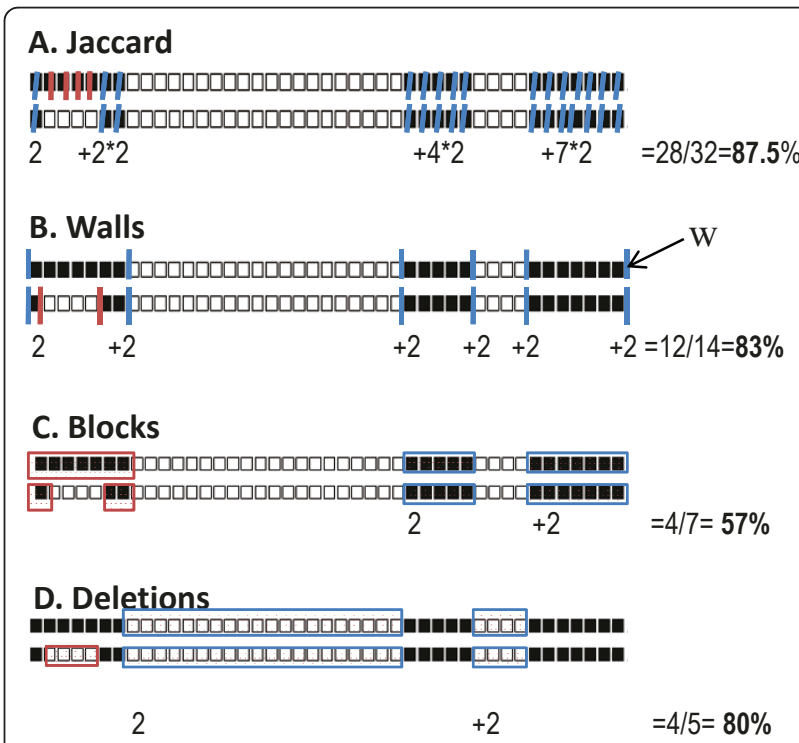

Figure 1 Distance methods. A: classically implemented Jaccard index. B-D: newly proposed distance methods. $\mathbf{w}=$ Domain Walls also referred to as walls. Numbers below the spoligotype patterns count the number of their common features: either the number of common spacers (A), common walls (B), common blocks (C), or common deletions (D). These numbers are summed and divided by the total number of features in the two spoligotype patterns to obtain the similarity between the two spoligotypes. 


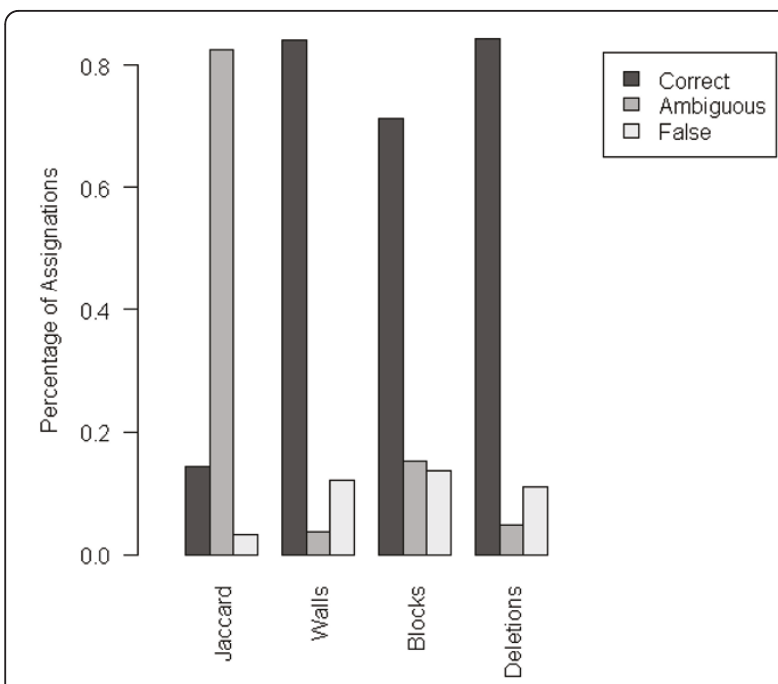

Figure 2 Assignations matches between SpolDB4 and the different distance methods on whole SpolDB4 database $(n=$ 1937 SIT). References are those described in Table 1. Assignations were performed according to the reference for which the distance was the lowest. The patterns for which the most similar reference is the same as that indicated by its SpolDB4 assignations, were scored as "Correct". Note that "Domain Walls" and "Deletions" have equally high values of assignations agreeing with the expert classification. When the method identified two identically similar references for a pattern, this pattern was scored as Unassigned and described as Ambiguous assignation. Ambiguity was the lowest with "Domain Walls" method.

the ten families. Spoligotype patterns for which two references were equally similar were coined as Unassignable. These assignations were compared to the SpolDB4 classification. The Jaccard method rarely associated the spoligotype patterns to their reference (only $12.3 \%$ of correctly assigned spoligotypes, see Figure 2). The methods that best fitted the expert classification were the "Deletions" (84.2\% of correctly assigned spoligotype patterns, $\mathrm{n}_{\text {correct }}=1402$ ), and the "Domain Walls" method $\left(84.0 \%, \mathrm{n}_{\text {correct }}=1399\right)$. These methods also provided the smallest amount of assignations that differed from those of the experts ("Deletions": $11.0 \%, \mathrm{n}_{\text {false }}=183$; "Domain Walls": $12.3 \%, \mathrm{n}$ false $=204$ ). These methods thus appear to be the best for fitting the expert classification out of the four methods we tested.

\section{"Deletions" method succeeds in correcting false SpolDB4 assignations}

Some families' assignations provided by SpolDB4 have been debated. For instance, patterns classified as LAM7TUR [32] have been found not to be related to the LAM family as strains carrying that pattern do not share the ligB ${ }^{1212}$ mutation that defines the LAM family [34]. Such strains were instead related to T-strains [35] and were renamed TUR. All methods tested here except the "Deletions" method still assigned them to LAM family, including Spotclust. The "Deletions" method assigned them all $\left(\mathrm{n}_{\text {LAM7-TUR }}=8\right)$ to the $\mathrm{T}$ family as did methods using VNTRs [35] or SNPs [34] (Table 2). Similarly, all spoligotypes assigned to the $\mathrm{H} 4$ subfamily $\left(n_{\mathrm{H} 4}=34\right)$ in SpolDB4 were recently excluded from the Haarlem family based on them not carrying the mgtC $\mathrm{C}^{545}$ mutation [34]. They were renamed "Ural" and appropriately assigned to the $\mathrm{T}$ family by the "Deletions" method only (Table 2). Hence, part of the assignations suspected to be wrong with the "Deletions" method as compared to expert classification may in fact correct previous classification errors. The "Deletions" method thus recognizes phylogenetic lineages better than "Domain Walls" method and likely at least as well as the expert eye and Spotclust. This is further supported by the clear gap between the similarity of correctly assigned spoligotype patterns to their reference (Figure 3D, black boxes in the Deletions plot) and the highest similarity to any reference of patterns assigned differently than by the expert (light gray boxes) specifically with the "Deletions" method.

Interestingly, Beijing, $\mathrm{X}$ and EAI families exhibited no incongruence between the "Deletions" and the expert method (no light gray box), suggesting that these families are clearly and appropriately defined. As reported above (Figure 2), the Jaccard method failed to assign most spoligotype patterns to any family; for instance, no spoligotype patterns could be assigned to BEIJ, EAI or $\mathrm{X}$ families (Figure $3 \mathrm{~A}$ ) with a maximum similarity to any reference not reaching $20 \%$ for BEIJ family (Additional file 1). "Domain Walls" and "Blocks" methods provided either poor resolution between correctly and wrongly assigned spoligotype patterns (Figure $3 \mathrm{~A}$ and $3 \mathrm{C}$ ), or a lower number of families with no discrepancy with the expert classification (only the $\mathrm{X}$ family with the "Domain Walls" method, Figure 3B).

\section{Assignations of $U$ spoligotype patterns}

Assignations thus seem phylogenetically relevant using the "Deletions" method and the references of the wellacknowledged families. We thus propose an alternative spoligotype patterns classification on the 1939 spoligotypes reported in SpolDB4 (Additional File 2). Assignation rate of "U" (Unclassified) patterns was relatively low with this method as compared to others (81 out of $272 \mathrm{U}$ patterns, i.e. $29.8 \%$, Figure 4 ) but may be more reliable as exemplified by three $U$ patterns recently assigned [34]: "Deletions" method could only assign one of them but without error whereas two of the three assignations provided by the "Domain Walls" method and Spotclust algorithm were erroneous (Table 2, SIT 105, 1274 and 1531). 
Table 2 Assignations of LAM7, H4 and selected "U" spoligotype patterns from SpolDB4, according to different methods.

\begin{tabular}{|c|c|c|c|c|c|c|c|c|}
\hline \multirow[b]{2}{*}{ SIT } & \multirow[t]{2}{*}{ spoligotype pattern } & \multicolumn{2}{|c|}{ SpolDB4 } & \multirow{2}{*}{$\begin{array}{c}\text { Recent litterature } \\
\text { assignation }\end{array}$} & \multirow{2}{*}{$\begin{array}{c}\text { Deletions } \\
\text { family }\end{array}$} & \multirow{2}{*}{$\begin{array}{c}\text { Domain Walls } \\
\text { family }\end{array}$} & \multicolumn{2}{|c|}{ SpotClust subfamily } \\
\hline & & family & Sub-family & & & & SpolDB3-based & RIM \\
\hline 41 & - & LAM & LAM7 & T-TUR & $T$ & LAM & LAM9 & N19 \\
\hline 186 & & LAM & LAM7 & T-TUR & $\mathrm{T}$ & LAM & LAM9 & N19 \\
\hline 367 & & LAM & LAM7 & T-TUR & $\mathrm{T}$ & LAM & LAM9 & N19 \\
\hline 930 & & LAM & LAM7 & T-TUR & $\mathrm{T}$ & LAM & LAMI & N19 \\
\hline 1261 & & LAM & LAM7 & T-TUR & $\mathrm{T}$ & LAM & LAM9 & N19 \\
\hline 1589 & & LAM & LAM7 & T-TUR & $\mathrm{T}$ & LAM & LAM3 & N2 \\
\hline 1924 & - & LAM & LAM7 & T-TUR & $\mathrm{T}$ & LAM & LAM9 & N19 \\
\hline 1937 & & LAM & LAM7 & T-TUR & $\mathbf{T}$ & LAM & LAM9 & N19 \\
\hline 35 & & $\mathrm{H}$ & $\mathrm{H} 4$ & T-Ural & $\mathrm{T}$ & $H$ & H3 & N34 \\
\hline 262 & . & $\mathrm{H}$ & $\mathrm{H} 4$ & T-Ural & $T$ & $H$ & H3 & N34 \\
\hline 361 & & $\mathrm{H}$ & $\mathrm{H} 4$ & T-Ural & $\mathbf{T}$ & $H$ & H3 & N34 \\
\hline 399 & & $\mathrm{H}$ & $\mathrm{H} 4$ & T-Ural & $\mathrm{T}$ & $H$ & $\mathrm{~T} 2$ & N34 \\
\hline 596 & & $\mathrm{H}$ & $\mathrm{H} 4$ & T-Ural & $T$ & $H$ & H3 & N34 \\
\hline 597 & 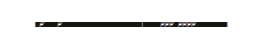 & $\mathrm{H}$ & $\mathrm{H} 4$ & T-Ural & $T$ & $H$ & $\mathrm{H} 3$ & N34 \\
\hline 656 & & $\mathrm{H}$ & $\mathrm{H} 4$ & T-Ural & $\mathrm{T}$ & $H$ & H3 & N34 \\
\hline 762 & & $\mathrm{H}$ & $\mathrm{H} 4$ & T-Ural & $\mathrm{T}$ & $H$ & H3 & N34 \\
\hline 777 & & $\mathrm{H}$ & $\mathrm{H} 4$ & T-Ural & $T$ & $H$ & H3 & N34 \\
\hline 817 & & $\mathrm{H}$ & $\mathrm{H} 4$ & T-Ural & $\mathrm{T}$ & $H$ & H3 & N34 \\
\hline 920 & & $\mathrm{H}$ & $\mathrm{H} 4$ & T-Ural & $\mathrm{T}$ & $H$ & H3 & N34 \\
\hline 921 & & $\mathrm{H}$ & $\mathrm{H} 4$ & T-Ural & $\mathrm{T}$ & $H$ & H3 & N34 \\
\hline 922 & & $\mathrm{H}$ & $\mathrm{H} 4$ & T-Ural & $\mathrm{T}$ & $H$ & $\mathrm{H} 3$ & N34 \\
\hline 1117 & & $\mathrm{H}$ & $\mathrm{H} 4$ & T-Ural & $\mathrm{T}$ & $H$ & H3 & N34 \\
\hline 1134 & & $\mathrm{H}$ & $\mathrm{H} 4$ & T-Ural & $\mathbf{T}$ & $H$ & H3 & N34 \\
\hline 1165 & & $\mathrm{H}$ & $\mathrm{H} 4$ & T-Ural & $T$ & $H$ & H3 & N34 \\
\hline 1174 & & $\mathrm{H}$ & $\mathrm{H} 4$ & T-Ural & $T$ & $H$ & $\mathrm{H} 3$ & N34 \\
\hline 1242 & & $\mathrm{H}$ & $\mathrm{H} 4$ & T-Ural & $\mathrm{T}$ & $H$ & U & N34 \\
\hline 1269 & & $\mathrm{H}$ & $\mathrm{H} 4$ & T-Ural & $\mathrm{T}$ & $H$ & H3 & N34 \\
\hline 1276 & & $\mathrm{H}$ & $\mathrm{H} 4$ & T-Ural & $\mathrm{T}$ & $H$ & H3 & N34 \\
\hline 1281 & & $\mathrm{H}$ & $\mathrm{H} 4$ & T-Ural & $\mathrm{T}$ & $H$ & H3 & N34 \\
\hline 1292 & & $\mathrm{H}$ & $\mathrm{H} 4$ & T-Ural & $\mathrm{T}$ & $H$ & H3 & N34 \\
\hline 1447 & & $\mathrm{H}$ & $\mathrm{H} 4$ & T-Ural & $\mathrm{T}$ & $H$ & H3 & N34 \\
\hline 1448 & & $\mathrm{H}$ & $\mathrm{H} 4$ & T-Ural & $\mathbf{T}$ & $H$ & $\mathrm{H} 3$ & N34 \\
\hline 1457 & & $\mathrm{H}$ & $\mathrm{H} 4$ & T-Ural & $\mathrm{T}$ & $H$ & H3 & N34 \\
\hline 1568 & & $\mathrm{H}$ & $\mathrm{H} 4$ & T-Ural & $\mathbf{T}$ & $H$ & H3 & N34 \\
\hline 1581 & & $\mathrm{H}$ & $\mathrm{H} 4$ & T-Ural & $\mathbf{T}$ & $H$ & H3 & N34 \\
\hline 1384 & & $\mathrm{H}$ & $\mathrm{H} 4$ & T-Ural & $T$ & U & T3 & N40 \\
\hline 1446 & & $\mathrm{H}$ & $\mathrm{H} 4$ & T-Ural & $\mathrm{T}$ & U & H3 & N34 \\
\hline 1452 & & $\mathrm{H}$ & $\mathrm{H} 4$ & T-Ural & $\mathrm{T}$ & U & H3 & N34 \\
\hline 1455 & & $\mathrm{H}$ & $\mathrm{H} 4$ & T-Ural & $T$ & U & U & N34 \\
\hline 1456 & & $\mathrm{H}$ & $\mathrm{H} 4$ & T-Ural & $\mathrm{T}$ & U & H3 & N34 \\
\hline 1461 & & $\mathrm{H}$ & $\mathrm{H} 4$ & T-Ural & $\mathrm{T}$ & U & H3 & N34 \\
\hline 1480 & & $\mathrm{H}$ & $\mathrm{H} 4$ & T-Ural & $\mathbf{T}$ & U & LAM9 & N19 \\
\hline 105 & - & $U$ & U & $\mathrm{H}$ & U & Afri & LAM7 & N29 \\
\hline 1274 & - & U & $U$ & LAM & U & Afri & H1 & N5 \\
\hline 1531 & - & $U$ & U & $x$ & $x$ & $\mathrm{x}$ & $\mathrm{X} 1$ & N44 \\
\hline
\end{tabular}

"Recent literature assignation" represents the standard, and refers to studies using loci other than the CRISPR locus: T-TUR classification has been suggested both by Millet et al. [35] and Abadia et al. [34] based respectively on VNTR signature and SNPs signatures. T-Ural classification has been suggested by Kovalev et al. [36] as they clustered with H37Rv strains and Abadia et al. [34]. RIM: Randomly Initialized Model. N ... families as defined by Spotclust are described on their website based on what SpolDB4 families/sub-families are mostly represented: N2 family is described as LAM3-rich; N5 as H1-rich; N19 as LAM-rich; N29 as LAM+EAIrich; N34 as H3+S-rich; N40 as T3-africanum-rich; N4 as X1-H37Rv-rich. The assignations matching the standard are shown in bold characters. Assignations failures are shown in italic. Note that the "Deletions" method provides the highest number of exact assignations and the least assignation failures. 


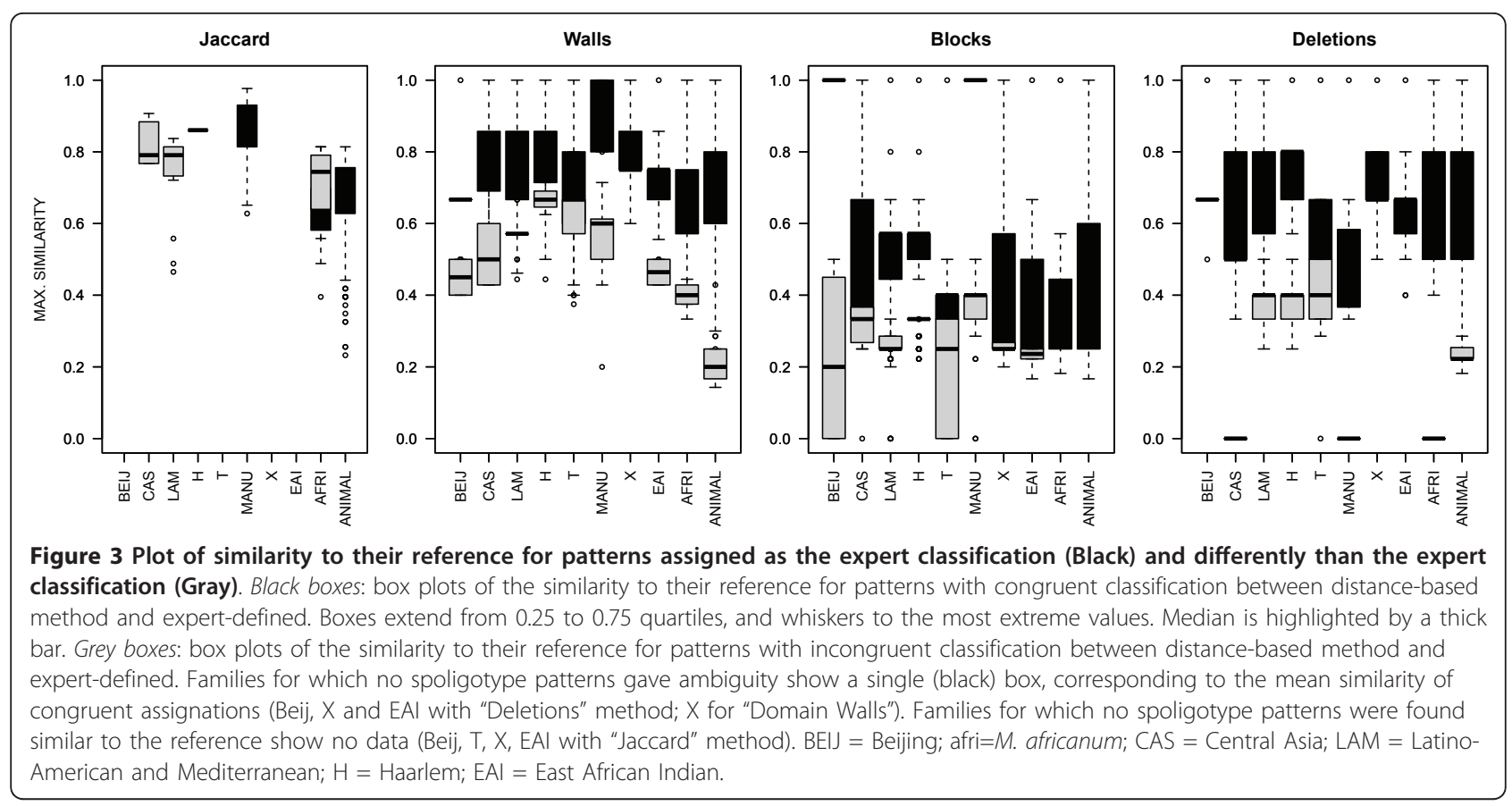

\section{Automatized identification of references by Affinity Propagation clustering}

The "Deletions" method is highly useful to classify spoligotype patterns in the described families, but this classification highly depends on the identification of references. These references are widely acknowledged

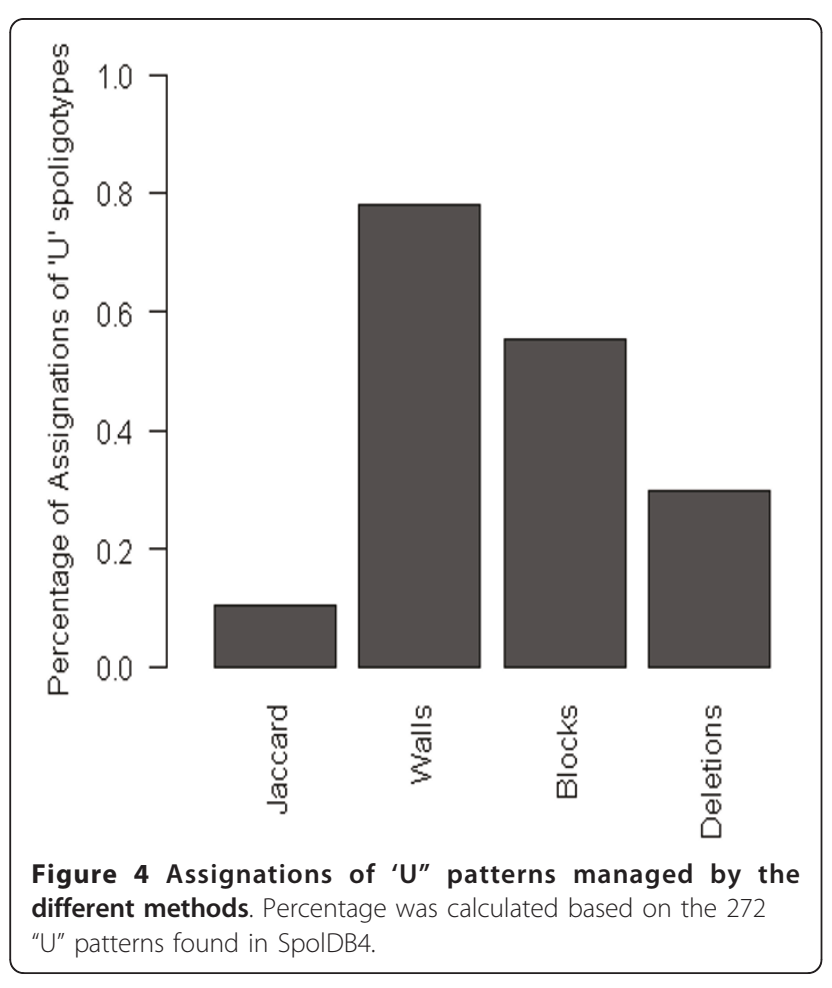

for major families but the relevance of finer classification is recurrently debated $[25,27,35]$. Affinity propagation is an algorithm that identifies a representative (also called exemplar) for each data point in an iterative manner until the chosen configuration of exemplars minimizes a suitable cost function that depends on the choice of the clusters (see Methods). A parameter set by the user (that we denote as 'penalty', $p$ ) determines an additional cost for every exemplar found. When $p$ is low (high negative value), large clusters are built where some data points have relatively low similarity with their representative. As $p$ increases, the clusters reach smaller sizes so that they become

numerous, and the mean similarity with the representative increases. Interestingly, when the number of clusters does not vary even if the penalty changes, this indicates that the data points are not evenly distributed, i.e., form meaningful clusters. When applying this method to the SpolDB4 dataset, relevant numbers of clusters were found to be 14 and 32 (Figure 5). The mean similarity with the representative was higher using Affinity Propagation as compared to K-Means or with Bionumerics applying hierarchical clustering (Additional File 3). The clustering in 14 clusters reproduced most of the 10 references identified by the experts (references for animal strains, CAS, EAI, H, LAM, T, and X, Table 3). However, $\mathrm{H}$ family was divided in $\mathrm{H} 1$ and $\mathrm{H} 3$, none of them including the H4. H4 was grouped with T spoligotype patterns as suggested by previous studies that renamed it Ural $[34,36]$. We propose some renamings according to major families represented in each cluster 


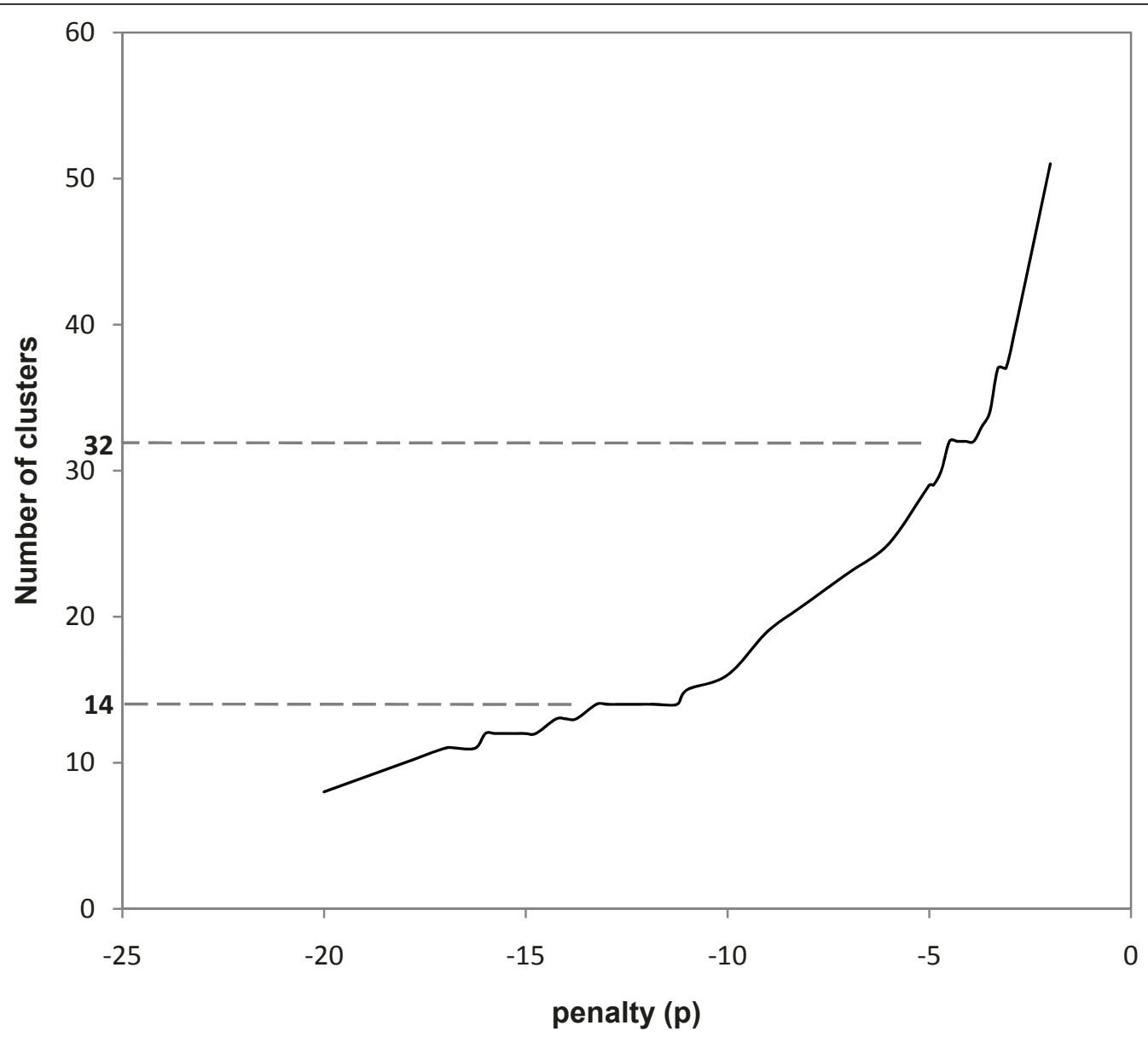

Figure 5 Number of clusters found by Affinity Propagation as a function of the penalty $p$ for the distance between a data point and its reference. Note that two plateau can be detected, at 14 and 32 clusters respectively, indicating that the corresponding clustering is robust, and therefore might be relevant.

Table 3 References after Affinity Propagation clustering for $\mathbf{n}_{\text {clusters }}=12$.

\begin{tabular}{|c|c|c|c|c|c|c|}
\hline AP-family & Reference & & & & Majoritary SpoIDB4 family & \\
\hline & SpolDB4 subfamily & SIT & Spoligotype pattern & Family & Proportion in the AP-family & Total Nb \\
\hline animal1 (Bov1-3-Cap-Mic-Pin) & bovis_1 & 482 & & Animal & 0.888 & 206 \\
\hline animal2(Bov2) & bovis_2 & 683 & & Animal & 0.621 & 66 \\
\hline Beij-afri & BEIJ & 255 & & Afri & 0.339 & 56 \\
\hline CAS & CAS_1 & 26 & & CAS & 0.760 & 96 \\
\hline EAI & EAI_5 & 236 & & EAl & 0.84 & 250 \\
\hline $\mathrm{H} 1-2$ & H_1 & 47 & & $\mathrm{H}$ & 0.853 & 68 \\
\hline $\mathbf{H} 3$ & H_3 & 50 & & $\mathrm{H}$ & 0.874 & 111 \\
\hline $\operatorname{LAM}_{(9-3-11-6-4)}$ & LAM_9 & 42 & & LAM & 0.721 & 179 \\
\hline $\mathrm{T} 2$ & T_2 & 52 & & T & 0.545 & 145 \\
\hline T3-LAM $(2-5)$ & LAM_2 & 17 & & LAM & 0.432 & 148 \\
\hline T-(Ural-H3-LAM $\left.{ }_{10-7)}\right)$ & T_1 & 53 & & $\mathrm{~T}$ & 0.823 & 351 \\
\hline$S(\& U)$ & S & 34 & & $\mathrm{~T}$ & 0.554 & 74 \\
\hline $\mathrm{T}(\& U)$ & T_1 & 173 & & $\mathrm{~T}$ & 0.420 & 81 \\
\hline$x$ & X_1 & 119 & 一 & $x$ & 0.75 & 108 \\
\hline
\end{tabular}

BEIJ = Beijing (also East Asia); afri=M. africanum; CAS = Central Asia (also East Africa and India); LAM = Latino-American and Mediterranean; $\mathrm{H}=\mathrm{Haarlem}$; EAI = East African Indian. Confirmed families are shown in bold. 
Table 4 References after Affinity Propagation clustering for $\mathbf{n}_{\text {clusters }}=32$.

\begin{tabular}{|c|c|c|c|c|c|c|c|}
\hline \multirow{3}{*}{$\begin{array}{c}\text { AP-subfamily } \\
\text { naming }\end{array}$} & \multicolumn{3}{|c|}{ reference } & \multicolumn{3}{|c|}{ Most represented subfamilies } & \multirow{3}{*}{$\begin{array}{c}\mathrm{Nb} \text { of spoligotype } \\
\text { patterns }\end{array}$} \\
\hline & \multirow[t]{2}{*}{$\begin{array}{c}\text { Classical subfamily } \\
\text { naming }\end{array}$} & \multirow[t]{2}{*}{ SIT } & \multirow[t]{2}{*}{$\begin{array}{l}\text { spoligotype (43 } \\
\text { format) }\end{array}$} & \multicolumn{2}{|c|}{$\begin{array}{l}\text { First most } \\
\text { represented } \\
\text { subfamily }\end{array}$} & \multirow[t]{2}{*}{$\begin{array}{l}\text { Second most repr. } \\
\text { family }\end{array}$} & \\
\hline & & & & Subfamily & Prop. & & \\
\hline Afri1 & AFRI1 & 181 & - & AFRI1 & 0.531 & AFRI & 32 \\
\hline Afri2-3 & AFRI2 & 331 & - & AFRI2 & 0.364 & AFRI3 & 22 \\
\hline Beij & BEIJ & 1 & & BEIJ & 0.842 & U & 19 \\
\hline Bov1-3 & BOV1 & 482 & - & BOV1 & 0.585 & $\mathrm{BOV}$ & 159 \\
\hline Bov2 & BOV2 & 683 & - & BOV2 & 0.467 & $\mathrm{BOV}$ & 45 \\
\hline Cap & CAP & 647 & & CAP & 0.75 & U & 20 \\
\hline CAS & CAS1 & 26 & & CAS1 & 0.487 & CAS & 80 \\
\hline EAI1 & EAl1 & 48 & 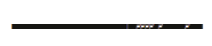 & EAl1 & 0.804 & U & 46 \\
\hline $\begin{array}{c}\text { EAI3-5 (del2-3-37- } \\
38-39)\end{array}$ & EAI2 & 11 & & EAl5 & 0.383 & EAl3 & 55 \\
\hline EAI2 (del3-20-21) & EAI2 & 19 & & EAI2 & 0.5 & $U$ & 48 \\
\hline EAI & EAI5 & 236 & & EAI5 & 0.651 & EAI4 & 86 \\
\hline EAI6 (del23-37) & EAl6 & 292 & & EAl6 & 0.5 & EAl5 & 42 \\
\hline $\mathrm{H} 1-2$ & $\mathrm{H} 1$ & 47 & & $\mathrm{H} 1$ & 0.790 & U & 62 \\
\hline H3 & $\mathrm{H} 3$ & 50 & & $\mathrm{H} 3$ & 0.927 & U & 96 \\
\hline Ural & $\mathrm{H} 4$ & 262 & & $\mathrm{H} 4$ & 0.714 & U & 28 \\
\hline LAM5-2-1(del3-13) & LAM2 & 17 & & LAM5 & 0.207 & U & 92 \\
\hline LAM3 & LAM3 & 33 & & LAM3 & 0.455 & U & 33 \\
\hline LAM & LAM9 & 42 & & LAM9 & 0.574 & LAM11 & 136 \\
\hline Manu & MANU2 & 54 & & MANU2 & 0.793 & U & 29 \\
\hline Pin-Mic & PIN & 637 & & $\mathrm{BOV}$ & 0.391 & U & 23 \\
\hline$S$ & S & 34 & & $\mathrm{~s}$ & 0.678 & U & 59 \\
\hline T (T1-H3-Lam10-Cam) & $\mathrm{T} 1$ & 53 & & $\mathrm{~T} 1$ & 0.828 & $\mathrm{H} 3$ & 261 \\
\hline T1a (del5-40-43) & $\mathrm{T} 1$ & 833 & & $\mathrm{~T} 1$ & 0.484 & U & 31 \\
\hline $\mathrm{T} 1 \mathrm{~b}$ (del21) & $\mathrm{T} 1$ & 291 & & U & 0.367 & $\mathrm{~T} 1$ & 30 \\
\hline T1c (del15) & $\mathrm{T} 2$ & 118 & & $\mathrm{~T} 1$ & 0.432 & U & 37 \\
\hline T2 (del40) & $\mathrm{T} 2$ & 52 & & $\mathrm{~T} 2$ & 0.521 & U & 119 \\
\hline T3 (del13) & $\mathrm{T} 3$ & 37 & & $\mathrm{~T} 3$ & 0.373 & U & 59 \\
\hline $\begin{array}{c}\text { T4 (del19-23-24-38- } \\
\text { 39) }\end{array}$ & $\mathrm{T} 4$ & 39 & & $\mathrm{~T} 1$ & 0.406 & $\mathrm{~T} 4$ & 32 \\
\hline T5 (del23) & T5 & 44 & & T5 & 0.561 & U & 41 \\
\hline$x$ & $\mathrm{X} 1$ & 119 & - & $\mathrm{X} 1$ & 0.492 & U & 61 \\
\hline $\mathrm{x} 2$ & $x_{2}$ & 137 & 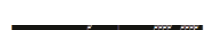 & $x_{2}$ & 0.824 & $\mathrm{~T} 1$ & 34 \\
\hline SEA1 (del29-34) & U & 458 & - & U & 0.955 & CAS & 22 \\
\hline
\end{tabular}

BEIJ = Beijing (also East Asia); afri=M. africanum; CAS = Central Asia (also East Africa and India); LAM = Latino-American and Mediterranean; $\mathrm{H}=\mathrm{Haarlem}$; EAI = East African Indian. Confirmed subfamilies are shown in bold.

(Table 3). When performing clustering with 32 clusters, many of the SpolDB4 subfamilies were identified. Some of them were however merged such as africanum2-africanum3, Bovis1-3, pinnipedii-microtii, LAM2-LAM1LAM5, X1 and 3 in X, etc. (Table 4). Among EAI, four meaningful subfamilies were identified whereas only 2 were among LAM. This suggests that the LAM family was oversplitted in the expert classification. In contrast, seven subfamilies were found among the $\mathrm{T}$ family. Two of them exhibited complex signatures with few spoligotype patterns actually matching the whole signature (for example, $n=5 / 29$ among T1a). Subfamilies T2, T3 and T5 were confirmed by this method. One family still had SIT53 (T1) as a reference indicating that many spoligotypes $(n=261)$ still cannot be further classified according to their spoligotype pattern. Last, one family was created from U spoligotypes and has SIT 458 as a reference. Most patterns carried the deletion of spacers 29 to 34 that could constitute a new significant signature. Countries in which the corresponding SITs 
Table 5 Spoligotype patterns clustered with SIT 458 with Affinity Propagation when $\mathbf{n}_{\text {clusters }}=32$.

\begin{tabular}{|c|c|c|c|}
\hline SIT & Spoligotype pattern & SpolDB4 assignation & Main country \\
\hline $458^{*}$ & . & $U$ & THA \\
\hline 354 & & $U$ & GBR \\
\hline 526 & & U & GNB \\
\hline 527 & & U & GNB \\
\hline 863 & & U & BRA \\
\hline 1172 & & U & EST \\
\hline 1186 & & U & THA \\
\hline 1187 & & U & THA \\
\hline 1374 & & U & MYS \\
\hline 1386 & & U & $B G D$ \\
\hline 1436 & & $U$ & $B G D$ \\
\hline 1462 & & U & GEO \\
\hline 1515 & & U & MDG \\
\hline 1518 & & $U$ & MDG \\
\hline 1519 & & $U$ & MDG \\
\hline 1520 & & U & MDG \\
\hline 1521 & & U & MDG \\
\hline 1524 & & U & MDG \\
\hline 710 & & U & NLD \\
\hline 405 & & U & VNM \\
\hline 426 & & CAS_2 & USA \\
\hline 523 & $\longrightarrow$ & U & MYS \\
\hline
\end{tabular}

Note that most of the patterns carry the 29-34 spacers deletion, and that most of them are unclassified by SpolDB4. "Main country" refers to the country where the highest number of isolates carrying this pattern were found according to SpolDB4 [33]. * indicates the spoligotype proposed as a reference by the Affinity Propagation algorithm. The countries are identified via the ISO3166-1 alpha-3 code. THA = Thailand; GBR = United Kingdom; GNB = Guinea-Bissau; BRA = Brazil; EST = Estonia; MYS = Malaysia; BGD = Bangladesh; GEO = Georgia; $\mathrm{MDG}=$ Madagascar; NLD $=$ Netherlands; VNM = Vietnam; USA = United States.

were most abundant surround the Indian Ocean: Madagascar, Thailand, India and Vietnam (Table 5). We thus named it SEA1 (South East Asia 1) (Table 4). The significance of this signature compared to the classical EAI signature, which differs only by the presence of spacer 33 , remains to be established.

\section{Discussion}

Here we first validated a simple distance method that can be used to classify CRISPR genetic profiles based on a worldwide $M$. tuberculosis spoligotype database. Second, using a recent clustering algorithm exploiting a different approach with respect to those commonly used in the biological sciences community, we could identify an alternative $M$. tuberculosis classification. The comparison between the largely validated expert classification described in the international database SpolDB4 and our alternative classification validates our approach for $M$. tuberculosis CRISPR profiles, opening the way for its use for other bacterial species where CRISPR were shown to provide interesting typing information [16].

\section{Clustering power of CRISPR patterns}

M. tuberculosis complex (MTC) has been infecting humans for at least 2600 years [37] and could be 20,000 years old or even much older [6,38]. Despite its restricted genetic diversity even between human and animal strains $[39,40]$, phylogenetical relationships have been detected using polymorphic DNA sequences $[41,42]$. CRISPR loci characterized using the "spoligotyping" technique have been used to define families through the use of so-called signatures i.e. the absence and presence of characterized units of CRIPSR loci, the spacers [43]. Most of these families found independent support such as host range or congruence with independent genetic markers [22,23,44], even SNPs and Regions of Deletion $[26,34,45]$. However, some of them were "illdefined" i.e. had a signature that was shared by several other groups, and others were defeated by independent loci: H4 subfamily was renamed Ural as it was related to $\mathrm{T}$ strains and not $\mathrm{H}$ strains [34,36], LAM7 and LAM10 were renamed TUR and Cameroon respectively as they are unrelated to LAM strains $[6,34,35]$. As a consequence, the use of CRISPR patterns to infer phylogenetical relationship was recurrently discussed $[44,46]$.

We used here an automatized approach for clustering CRISPR patterns. Our clusters largely reproduced the well-acknowledged MTC families and provided meaningful clustering for Ural, TUR and Cameroon. In fact, the misclassification of Ural among Haarlem family was due to the merging of all signatures having spacer 31 deleted and spacer 32 present disregarding the left border of the deletion. This classification criterion is not relevant knowing the evolutionary dynamics of CRISPR loci due either to the insertion of IS6110 elements or to the deletion of one of several adjacent spacers. This kind of errors is avoided if comparison is performed using an algorithm identifying complete signatures (left and right borders of the deletions) as included in our automatized approach (see below for a detailed discussion on methods used to calculate distances between strains).

Still, the fact that some families are "ill-defined" is an intrinsic problem of spoligotyping: CRISPR loci in $M$. tuberculosis are relatively short and they evolve at a rate that cannot exclude the absence or the insufficient number of mutation in some lineages. This intrinsically limits the power of our study, i.e. we cannot classify all strains, and not all of them with the same precision. However, this problem does not affect the assignation quality of the strains we classify which are in fact numerous (more than 80\%).

We thus argue that CRISPR profiles evolving by the insertion of transposable elements or by deletion such as those of $M$. tuberculosis contain relevant information for clustering and even inference of some phylogenetic 
links. The targeted locus must however not be missing for the individuals to be classified. To avoid this pitfall, the use of CRISPR loci should restrict to recently diverged groups as is the M. tuberculosis species complex (more than 99.9\% identity). Such organisms uncover diverse human pathogens such as Yersinia pestis, Salmonella enterica, Bacilllus anthracis, Mycobacterium leprae and Mycobacterium ulcerans. Still, the use of CRISPR profiles in phylogenetic reconstructions would benefit from further developments and validations for species with still active CRISPR loci.

\section{Distance methods for CRISPR profiles}

If CRISPR can be used to infer phylogenetic relationship, the evolutionary model or distance method used during the inference is also of great importance. Several developments had been proposed until now. We want to discuss here what our approach adds to previous ones.

CRISPR profiles (spoligotype patterns) form a sequence of binary data. As such, it has been analyzed with tools developed for binary information such as the Jaccard Index that focuses on the sharing of every unit in the profile (here the spacers) taken independently. This however ignores an essential feature of the corresponding CRISPR locus: that it evolves by the loss of spacers. These losses can occur either because of the insertion of a transposable element that disrupts the sequence used in the spoligotyping technique, or by deletion. Deletions can occur for several spacers at once, even if the frequency of large deletions may be lower [21]. As a consequence, the distance between two patterns, one carrying many spacers and the other carrying one large deletion, should not be considered as proportional to the number of spacers that were lost (as done by the Jaccard index), but as corresponding to a single mutation event. The methods proposed by the Bennett laboratory $[30,31]$ take into account the deletion process and add a probability function that best mimics the frequency of deletion size. In Spotclust, a Bayesian algorithm incorporating the inference of ancestral spoligotype patterns based on SpolDB3 database is used to assign spoligotypes to SpolDB3 subfamilies or to families built using a Randomly Initialized Model (RIM) [30]. We showed here that, by simply using expertdefined references of main families and the "Deletions" distance method that is based on deletion signatures, we could better assign Unknown spoligotype patterns than Spotclust as well as correct previous erroneous assignations in SpolDB4 classification such as those to LAM7 (TUR) [29]. For Spotclust algorithm, this was true for both the SpolDB3-based classification and the Randomly Initialized Model. The reason for that could be either that the size of the database used by Spotclust was too small to capture evolutionary steps relevant to MTC evolution, or that Bayesian statistical inferences are too dependent on priors.

\section{Performance of the Affinity propagation algorithm on CRISPR profiles clustering}

Affinity Propagation is a message-passing algorithm that considers clustering as a problem of minimizing an "energy" function of the clusters configuration in the data set (see Methods section for a general review of the algorithm, and [8]). This approach seems particularly promising and could help solving species delineations in asexual lineages where obligate gene exchange cannot be used as a delineation criterion [9]. One of the main features of the algorithm is the possibility of regulating the total number of clusters as a function of an input parameter of the algorithm (called the "chemical potential" $\mu$, by analogy to the chemical potential of physical systems, or $\mathrm{p}$ for penalty parameter, see Methods). Also the high speed (the computational time goes as $\mathrm{N}^{2}$ if $\mathrm{N}$ is the size of the dataset) and thus the possibility to analyze very large networks is encouraging the use of this algorithm. With this method we identified both families and subfamilies in MTC. A single family out of 14 made no sense (Beijing-africanum). This is likely due to a lack of information in Beijing spoligotype pattern as the large 1-36 deletion limits the recognition of other signatures. When considering patterns carrying a larger number of spacers, the classification was largely congruent with the literature. In addition, we could identify new signatures, especially one, termed SEA1, among previously unclassified patterns. We therefore believe that this algorithm is very useful for classifying the widely used 43-spoligotype patterns in $M$. tuberculosis but could prove even more useful on patterns larger than 43 spacers, e.g. the improved 68 spacers format.

\section{"Euro-american" lineage evolution}

Despite large sequencing efforts [25,47], there has been a standing difficulty in unraveling the relationships inside "Euro-American" lineage strains (carrying the 3336 deletion in the spoligotype pattern), especially in the so-called "T family" described in SpolDB4 [23]. Here, using SpolDB4 database, we could challenge expertdefined families and subfamilies. We first confirmed the validity of $\mathrm{S}$ and $\mathrm{T} 2$ subfamilies that we suggest to consider as families. The $\mathrm{S}$ family was first described in Sicily [48] and independently identified in Québec where a sublineage was shown to harbor a peculiar pncA SNP [49]. The T2 family, defined by the absence of spacer 40 was originally described as $M$. africanum 2 , however was shown later to be a bona fide M. tuberculosis subfamily [50]. We also confirmed the reliability of Haarlem family subclustering, if renaming $\mathrm{H} 4$ as Ural, 
and suggest considering $\mathrm{H} 1-2$ and $\mathrm{H} 3$ as two families. We confirmed the validity of T3 and T5 families as well as T4-CEU (although T4 alone was invalidated). Some LAM subfamilies renamings based on VNTR and SNP loci [34-36] are given further support (LAM7 as TUR, LAM10 as Cameroon), while other were merged (LAM1, 2 and 5). The tendency to merge many expertdefined families was not pervasive. Indeed in the EAI family, four subfamilies out of the 5 expert-defined ones were confirmed.

Combining the families and subfamilies identification, we could provide a simplified evolutionary scheme for this lineage (Figure 6). We hope in the future, by applying affinity propagation on 68 spoligotype patterns, to identify other Euro-American subclusters.

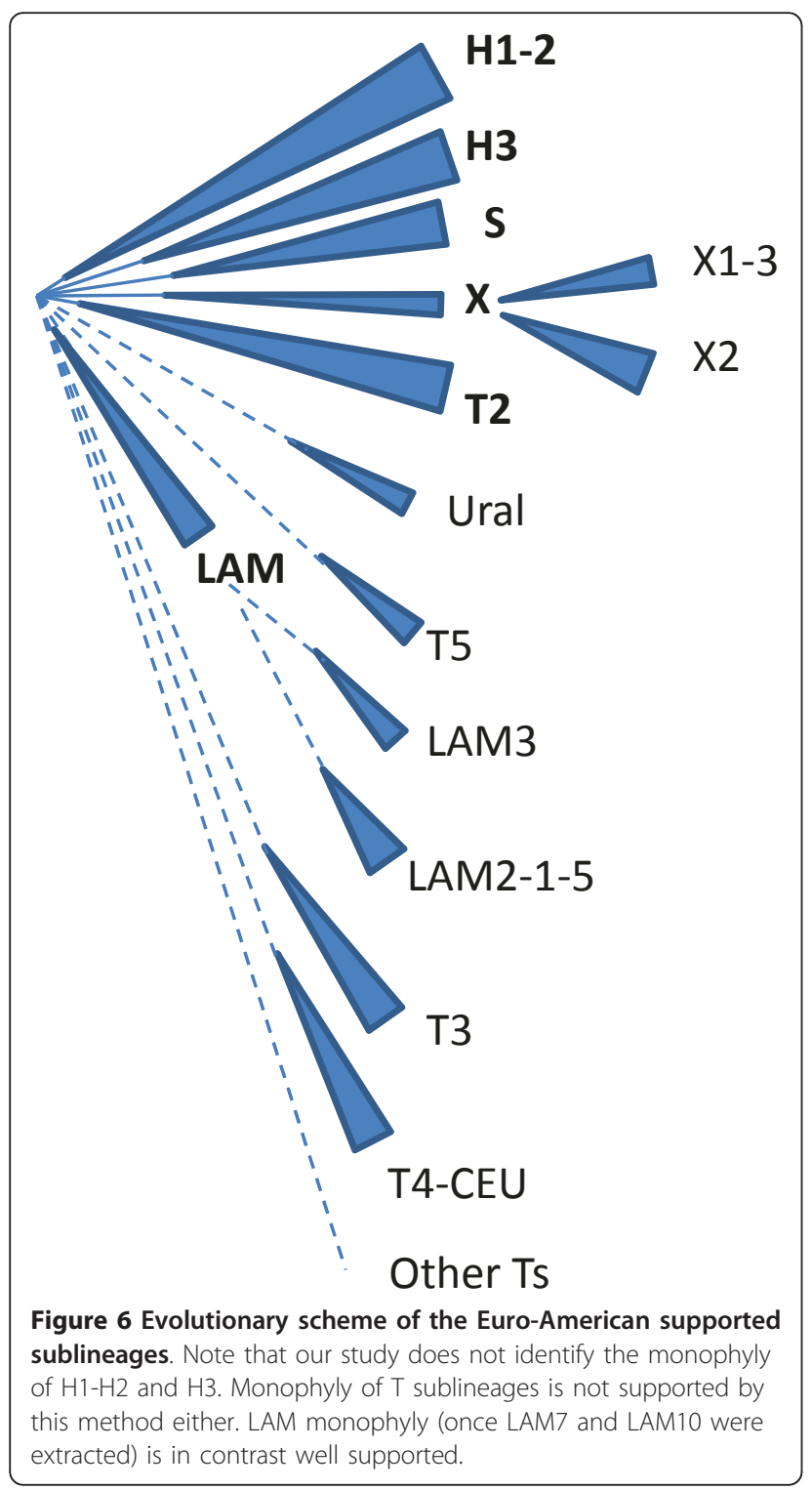

\section{Conclusion}

This study describes 1) a novel distance method to be applied on genetic loci evolving by deletion, as for instance do inactive CRISPR loci, 2) a framework to take advantage of identified references for classifying individuals using such loci, 3) a way to identify new references using the Affinity Propagation algorithm [8], and 4) assignations and assignation tools for M. tuberculosis complex. The distance method and the framework to identify known references were largely validated by worldwide $M$. tuberculosis database at the CRISPR locus (spoligotype patterns). This work encourages the use of CRISPR patterns to cluster strains in other organisms carrying such loci and for which wide genotyping has been undertaken as it is now the case for human pathogens such as Yersinia pestis, Salmonella enterica, Bacilllus anthracis, Mycobacterium leprae and M. ulcerans. Affinity propagation could also be useful to cluster other genotyping data such as SNPs or minisatellites. Databases larger than those available by now are however required to test the validity of this method on such markers.

\section{Methods}

\section{Spoligotyping data}

SpolDB4[33], containing 1939 shared international spoligotypes (SIT) was used as raw data for spoligotyping diversity analysis. This database contains family or subfamily information, with some uncertainties indicated such as LAM3 and S-convergent or T1-T2. To simplify the analyses, when two assignations were provided, only the first one was kept. We also merged certain families when the number of spoligotype patterns was very small and not been confirmed by SNP typing [40]. Specifically, the families we retained are: africanum $(n=46)$, animal strains (grouping BOVIS, PINNIPEDII, MICROTII, CAPRAE, $\left.n_{\text {tot }}=231\right)$, Beijing $(n=21)$, CAS $(n=86)$, EAI $(\mathrm{n}=213), \mathrm{H}(\mathrm{n}=233)$, LAM $(\mathrm{n}=224)$, MANU $(\mathrm{n}$ $=39), \mathrm{T}(\mathrm{n}=482)$ including $\mathrm{S}$ and H37Rv ST as suggested by Brudey et al (2006), X $(\mathrm{n}=90)$. We excluded SIT69 which was suppressed by Institut Pasteur [33] as well as the canetti spoligotype pattern which is unique (SIT592). There are 272 unclassified spoligotypes (U). The references for each family correspond to SpolDB4 description [23]; they are listed in Table 1.

\section{Methods to compute distances}

Three new methods to compute distances were designed that fit CRISPR loci evolutionary dynamics such as that of $M$. tuberculosis complex i.e. evolution by deletion or transposon insertion. All methods rely on the identification of the beginning and the end of spacers deletions. These limits were named Domain Walls (Figure 1). The 
"Domain Walls" method measures the proportion of common Domain Walls between two CRISPR profiles, $i$. $e$. if the profiles have $i_{\mathrm{w}}$ and $\mathrm{j}_{\mathrm{w}}$ Domain Walls respectively and $K_{w}$ are common, the distance is:

$$
d_{D W a l l s}=\frac{2 K_{w}}{i_{w}+j_{w}}
$$

The "Blocks" method considers blocks of spacers; let $i_{b}$ and $j_{b}$ be the numbers of blocks carried by the two profiles and $K_{b}$ the number of blocks they share,

$$
d_{\text {Blocks }}=\frac{2 K_{b}}{i_{b}+j_{b}}
$$

The "Deletions" method considers deleted blocks; let $i_{d}$ and $j_{d}$ be the numbers of blocks of deletions carried by the two profiles and $K_{d}$ the number of shared deletions,

$$
d_{\text {Deletions }}=\frac{2 K_{d}}{i_{d}+j_{d}}
$$

These distance methods were used to compute the distance between each SpolDB4 spoligotype pattern and the references of the ten main $M$. tuberculosis complex families. The scripts for such calculations were written in R language [51] and are available upon request. Each pattern was assigned to the family whose reference it was the most similar to.

\section{Clustering algorithms}

Affinity Propagation (AP), proposed first in [8], is a recent clustering method based on the choice of "exemplars" as centers of the clusters, i.e., one representative data point for each cluster to which the other nodes rely. The choice of the exemplars is based on the minimization of the total "energy" of the system, function of the total distance between data points and exemplars in a given clusters configuration. This method falls in the class of message-passing type algorithms, exploiting the Belief Propagation method (also known as Cavity method in the physics literature) to minimize the energy function in an computationally efficient way (from the exponential time complexity of the naïve methods to $\mathrm{O}$ $\left(\mathrm{N}^{2}\right)$, where $\mathrm{N}$ is the total number of nodes to cluster). The starting point is thus a set of data points, representing the nodes of the network, and a similarity matrix $\mathrm{S}$ defining the similarities among all the nodes as deduced from the distance between all these nodes. The similarity between two points $i$ and $j$ is defined as

$$
S(i, j)=1-d(i, j)
$$

provided that the distance $d$ ranges from 0 to 1 , as in our case (this is always true up to a normalization of the distance). The aim is then to find a map $c:\{1, \ldots, N\}$ $\rightarrow\{1, \ldots, N\}$, with $N$ being the total number of data points and $c(i) \equiv c_{i}$ is the exemplar of node $i$, such that the vector $\bar{c}=\left(c_{1}, \ldots, c_{N}\right)$ minimizes the energy function

$$
E(\bar{c})=-\sum_{i=1}^{N} S\left(i, c_{i}\right)-\frac{1}{\beta} \sum_{i=1}^{N} \log \left(\chi_{i}(\bar{c})\right)
$$

The first term of the function defined above is (minus) the sum of all the similarities between a point and its exemplar, while the second term is introduced to avoid any configuration in which an exemplar does not belong to the cluster that itself represents, that is, an exemplar must be the exemplar of itself. This is granted by defining the function $\chi_{i}(\bar{c})$ as

$$
\chi_{i}(\bar{c})=\left\{\begin{array}{l}
0 c_{i} \neq i \cap \exists j: c_{j}=i \\
1 \text { otherwise }
\end{array}\right.
$$

and by taking the log function of it and summing it over all the nodes, so that the energy becomes infinite if at least an exemplar is represented by a different exemplar. The parameter $\beta$ plays formally the role of the inverse of the temperature in a thermodynamical system, and thus determines the level of thermal noise acting on the system. This means that varying this parameter, but keeping it finite, allows the algorithm to accept configurations of the clusters that do not exactly corresponds to minima of the energy function. We consider here only the optimal case of zero temperature, $i$. $e ., \beta \rightarrow \infty$ (for a general and exhaustive treatment of the cavity method see, for example [52]).

Once the Cavity equations are written one is left with two coupled update rules for each couple of nodes $(i, j)$ :

$$
\begin{aligned}
& r_{i \rightarrow j}^{t+1}=S(i, j)-\max _{k \neq j}\left(a_{k \rightarrow i}^{t}+S(k, i)\right) \\
& a_{j \rightarrow i}^{t+1}=\min \left(0, r_{j \rightarrow j}^{t}+\sum_{k \neq i, j} \max \left(0, r_{k \rightarrow j}^{t}\right)\right)
\end{aligned}
$$

These update rules represents messages that the nodes are exchanging between iteration $t$ and $t+1$, with $r_{i \rightarrow j}^{t}$ and $a_{j \rightarrow i}^{t}$ representing respectively the energetic "competition" between node $i$ and all the other nodes except $j$ to be the exemplar of node $j$ and the gain in the total energy of the system if node $j$ is represented by node $i$. The notation $i \rightarrow j$ indicates that the message is sent from node $i$ toward node $j$. When the update equations converge, then the value of each $c_{i}, i=1, \ldots, N$, is obtained summing over all the messages arriving at node $i$ and maximizing the sum. The diagonal elements of the similarity matrix, that are not constrained to be equal to the unity, play the role of an effective cost to 
every chosen exemplar, and thus a cost for the total number of clusters found. They are a fine-tuning for the selection of the total number of clusters found by AP. In our study we chose to consider every data point $a$ priori equally probable to be an exemplar, so we set $S(i$, $i)=p \forall i=1, \ldots, N$. Varying the parameter $p$ from very large (negative) values up to positive values gives the range of total clusters from 1 to $\mathrm{N}$, and we interpret a stability in the total number of clusters under changes of this parameter as a genetically meaningful grouping of the data, as discussed in the Results section. The similarity matrix S was obtained using the "Deletions" distance that had turned out to be the most accurate distance. Linear combinations of the various distances introduced in the previous section were also considered, but the overall result still favors the Deletions distance. We performed also a comparison of AP with other "classical" clustering algorithms, such as K-Means and Hierarchical clustering. We considered the performance with respect to the experts' classification as defined in SpolDB4 $[23,33]$ and identified that AP found clusters with much lower error (see Additional File 3). The script for computing the distance matrices of SpolDB4 database and performing the analysis with AP was written in Matlab as a self-contained script, the bare AP algorithm for Matlab is available from the authors Frey and Dueck.

\section{Additional material}

Additional file 1: Plot of similarity to their reference for patterns assigned as the expert classification (Green), patterns not assigned due to ambiguity (Gray) and patterns assigned differently than the expert classification (Red).

Additional file 2: SpolDB4 new assignations, using the previously identified references or the newly identified ones.

Additional file 3: Mean similarity of patterns with their representative as a function of the cluster size, and for different clustering methods (AP: Affinity Propagation; Bio: Bionumerics; KM: K-Means).

\begin{abstract}
Acknowledgements
Marc Mézard is acknowledged for fruitful discussions on the design of the study. Edgar Abadia, Jian Zhang and Michel Gomgnimbou are acknowledged for discussions on the design of the manuscript. CS and SF acknowledge financial support of Univ. Paris-Sud in the form of a "Chaire d'excellence » respectively in Physics (SF) and Microbiology (CS).
\end{abstract}

\section{Author details}

'LPTMS, CNRS and Univ. Paris-Sud, UMR8626, Bat. 100, 91405 Orsay, France. 2Dipartimento di Fisica "G. Galilei", Università di Padova, via Marzolo 8, I35131 Padova, Italy. ${ }^{3}$ IGM, CNRS and Univ. Paris-Sud, UMR8621, Bat. 400, F91405 Orsay cedex, France. ${ }^{4}$ Unité de Génétique Mycobactérienne, Institut Pasteur, Paris, France.

\section{Authors' contributions}

CS and SF initiated this work through informal discussions; ML did the first experiments under SF supervision and CS guidance for classification
(University Paris-Sud Master 1 program, Physics). ML wrote his Master report on this topic. SF supervised CB (Master 2 program) to program writing, acquisition and analysis of data. GR performed complementary program writing, data acquisition and analysis. CB and GR were both involved in data analysis and writing of the manuscript. CS provided the taxonomic expertise and contributed to the revision of the manuscript. All authors approved the final version.

Received: 18 January 2011 Accepted: 2 June 2011

Published: 2 June 2011

\section{References}

1. Le Flèche P, Fabre M, Denoeud F, Koeck J, Vergnaud G: High resolution, on-line identification of strains from the Mycobacterium tuberculosis complex based on tandem repeat typing. BMC Microbiol 2002, 2:37.

2. Pourcel C, Salvignol G, Vergnaud G: CRISPR elements in Yersinia pestis acquire new repeats by preferential uptake of bacteriophage DNA, and provide additional tools for evolutionary studies. Microbiology 2005, 151(Pt 3):653-663.

3. Zhang J, Abadia E, Refregier G, Tafaj S, Boschiroli ML, Guillard B, Andremont A, Ruimy R, Sola C: Mycobacterium tuberculosis complex CRISPR genotyping: improving efficiency, throughput and discriminative power of 'spoligotyping' with new spacers and a microbead-based hybridization assay. J Med Microbiol 2009, 59((Pt 3)):285-94.

4. Deshpande A, Gans J, Graves SW, Green L, Taylor L, Kim HB, Kunde YA, Leonard PM, Li PE, Mark J, et al: A rapid multiplex assay for nucleic acidbased diagnostics. Journal of Microbiological Methods 2010, 80(2):155-163.

5. Pritchard JK, Stephens M, Donnelly P: Inference of population structure using multilocus genotype data. Genetics 2000, 155(2):945-959.

6. Wirth T, Hildebrand F, Allix-Beguec C, Wolbeling F, Kubica T, Kremer K, van Soolingen D, Rusch-Gerdes S, Locht C, Brisse S, et al: Origin, spread and demography of the Mycobacterium tuberculosis complex. PLOS Pathog 2008, 4(9):e1000160.

7. MacQueen J: Proceedings of the Fifth Berkeley Symposium on Mathematical Statistics and Probability 1967 Univ of California Press, Berkeley, CA; 1967.

8. Frey BJ, Dueck D: Clustering by passing messages between data points. Science 2007, 315(5814):972-976.

9. Bailly-Bechet M, Bradde S, Braunstein A, Flaxman A, Foini L, Zecchina R: Clustering with shallow trees. Journal of Statistical Mechanics-Theory and Experiment 2009.

10. Posada D, Crandall KA: MODELTEST: testing the model of DNA substitution. Bioinformatics 1998, 14:817-818.

11. Ellegren $\mathrm{H}$ : Microsatellites: Simple sequences with complex evolution. Nature Reviews Genetics 2004, 5(6):435-445.

12. Jansen R, van Embden JD, Gaastra W, Schouls LM: Identification of a novel family of sequence repeats among prokaryotes. Genomics 2002, 6(1):23-33.

13. Sorek R, Kunin V, Hugenholtz P: CRISPR-a widespread system that provides acquired resistance against phages in bacteria and archaea. Nat Rev Microbiol 2008, 6(3):181-186.

14. Barrangou R, Fremaux C, Deveau H, Richards M, Boyaval P, Moineau S, Romero DA, Horvath P: CRISPR provides acquired resistance against viruses in prokaryotes. Science 2007, 315(5819):1709-1712.

15. Garneau JE, Dupuis ME, Villion M, Romero DA, Barrangou R, Boyaval P, Fremaux C, Horvath P, Magadan AH, Moineau S: The CRISPR/Cas bacterial immune system cleaves bacteriophage and plasmid DNA. Nature 2010, 468(7320):67+

16. Liu F, Barrangou R, Gerner-Smidt P, Ribot EM, Knabel SJ, Dudley EG: Novel Virulence Gene and Clustered Regularly Interspaced Short Palindromic Repeat (CRISPR) Multilocus Sequence Typing Scheme for Subtyping of the Major Serovars of Salmonella enterica subsp enterica. Appl Environ Microbiol 2011, 77(6):1946-1956.

17. Mojica FJ, Diez-Villasenor C, Garcia-Martinez J, Soria E: Intervening sequences of regularly spaced prokaryotic repeats derive from foreign genetic elements. J Mol Evol 2005, 60(2):174-182.

18. Kamerbeek J, Schouls L, Kolk A, van Agterveld M, van Soolingen D, Kuijper S, Bunschoten A, Molhuizen H, Shaw R, Goyal M, et al: Simultaneous detection and strain differentiation of Mycobacterium tuberculosis for diagnosis and epidemiology. J Clin Microbiol 1997, 35(4):907-914.

19. Kremer $K$, van Soolingen D, Frothingham R, Haas WH, Hermans PW, Martin C, Palittapongarnpim P, Plikaytis BB, Riley LW, Yakrus MA, et al: 
Comparison of methods based on different molecular epidemiological markers for typing of Mycobacterium tuberculosis complex strains: interlaboratory study of discriminatory power and reproducibility. J Clin Microbiol 1999, 37(8):2607-2618

20. Tafaj S, Zhang J, Hauck Y, Pourcel C, Hafizi H, Zoraqi G, Sola C: First insight into genetic diversity of the Mycobacterium tuberculosis complex in Albania obtained by multilocus variable-number tandem-repeat analysis and spoligotyping reveals the presence of beijing multidrug-resistant isolates. J Clin Microbiol 2009, 47(5):1581-1584

21. Reyes JF, Francis AR, Tanaka MM: Models of deletion for visualizing bacterial variation: an application to tuberculosis spoligotypes. BMC Bioinformatics 2008, 9:496.

22. Filliol I, Driscoll JR, Van Soolingen D, Kreiswirth BN, Kremer K, Valétudie G, Anh DD, Barlow R, Banerjee D, Bifani PJ, et al: Global distribution of Mycobacterium tuberculosis spoligotypes. Emerg Inf Dis 2002, 8(11):1347-1349.

23. Brudey K, Driscoll J, Rigouts L, Prodinger WM, Gori A, Al-Hajoj SAM, Allix C, Aristimuno L, Arora J, Baumanis V, et al: Mycobacterium tuberculosis complex genetic diversity: mining the fourth international spoligotyping database (SpolDB4) for classification, Population Genetics, and Epidemiology. BMC Microbiol 2006, 6(6):23.

24. Sola C, Devallois A, Horgen L, Maisetti J, Filliol I, Legrand E, Rastogi N: Tuberculosis in the Caribbean: using spacer oligonucleotide typing to understand strain origin and transmission. Emerg Infect Dis 1999, 5(3):404-414.

25. Comas I, Homolka S, Niemann S, Gagneux S: Genotyping of Genetically Monomorphic Bacteria: DNA Sequencing in Mycobacterium tuberculosis Highlights the Limitations of Current Methodologies. Plos One 2009, 4(11):e7815

26. Zhang J, Abadia E, Refregier G, Tafaj S, Boschiroli ML, Guillard B, Andremont A, Ruimy R, Sola C: Mycobacterium tuberculosis complex CRISPR genotyping: improving efficiency, throughput and discriminative power of 'spoligotyping' with new spacers and a microbead-based hybridization assay. J Med Microbiol 2010, 59(Pt 3):285-294.

27. Gagneux S, DeRiemer K, Van T, Kato-Maeda M, de Jong BC, Narayanan S, Nicol M, Niemann S, Kremer K, Gutierrez MC, et al: Variable host-pathogen compatibility in Mycobacterium tuberculosis. Proc Natl Acad Sci USA 2006, 103(8):2869-2873.

28. Sreevatsan S, Pan X, Stockbauer KE, Connell ND, Kreiswirth BN, Whittam TS, Musser JM: Restricted structural gene polymorphism in the Mycobacterium tuberculosis complex indicates evolutionarily recent global dissemination. Proc Natl Acad Sci USA 1997, 94(18):9869-9874.

29. SpotClust. [http://www.rpi.edu/ bennek/EpiResearch].

30. Vitol I, Driscoll J, Kreiswirth B, Kurepina N, Bennett KP: Identifying Mycobacterium tuberculosis complex strain families using spoligotypes. Infect Genet Evol 2006, 6(6):491-504.

31. Aminian M, Shabbeer A, Bennett KP: A conformal Bayesian network for classification of Mycobacterium tuberculosis complex lineages. Bmc Bioinformatics 2010, 29(11):Suppl 3-S4.

32. Zozio T, Allix C, Gunal S, Saribas Z, Alp A, Durmaz R, Fauville-Dufaux M, Rastogi N, Sola C: Genotyping of Mycobacterium tuberculosis clinical isolates in two cities of Turkey: description of a new family of genotypes that is phylogeographically specific for Asia Minor. BMC Microbiol 2005, 5:44.

33. SpolDB4. [http://www.pasteur-guadeloupe.fr:8081/SITVITDemo/].

34. Abadia E, Zhang J, Vultos T, Ritacco V, Kremer K, Aktas E, Matsumoto T, Refregier G, Soolingen DV, Gicquel B, et al: Resolving lineage assignation on Mycobacterium tuberculosis clinical isolates classified by spoligotyping with a new high-throughput 3R SNPs based method. Infect Genet Evol 2010, 10(7):1066-1074.

35. Millet J, Miyagi-Shiohira C, Yamane N, Sola C, Rastogi N: Assessment of mycobacterial interspersed repetitive unit-QUB markers to further discriminate the Beijing genotype in a population-based study of the genetic diversity of Mycobacterium tuberculosis clinical isolates from Okinawa, Ryukyu Islands, Japan. J Clin Microbiol 2007, 45(11):3606-3615.

36. Kovalev SY, Kamaev EY, Kravchenko MA, Kurepina NE, Skorniakov SN: Genetic analysis of Mycobacterium tuberculosis strains isolated in Ural region, Russian federation, by MIRU-VNTR genotyping. Int I Tuberc Lung Dis 2005, 9(7):746-752.

37. Donoghue HD, Lee OYC, Minnikin DE, Besra GS, Taylor JH, Spigelman M: Tuberculosis in Dr Granville's mummy: a molecular re-examination of the earliest known Egyptian mummy to be scientifically examined and given a medical diagnosis. Proceedings of the Royal Society B-Biological Sciences 2010, 277(1678):51-56.

38. Gutierrez MC, Brisse S, Brosch R, Fabre M, Omais B, Marmiesse M, Supply P, Vincent $\mathrm{V}$ : Ancient origin and gene mosaicism of the progenitor of Mycobacterium tuberculosis. Plos Pathogens 2005, 1(1):e5.

39. Brosch R, Gordon SV, Pym A, Eiglmeier K, Garnier T, Cole S: Comparative genomics of the mycobacteria. Int J Med Microbiol 2000, 290:143-152.

40. Hershberg R, Lipatov M, Small PM, Sheffer H, Niemann S, Homolka S, Roach JC, Kremer K, Petrov DA, Feldman MW, et al: High functional diversity in Mycobacterium tuberculosis driven by genetic drift and human demography. PLOS Biol 2008, 6(12):e311.

41. van Soolingen D, de Haas PEW, Hermans PWM, Groenen PMA, van Embden JDA: Comparison of various repetitive DNA elements as genetic markers for strain differentiation and epidemiology of Mycobacterium tuberculosis. J Clin Microbiol 1993, 31:1987-1995.

42. Eisenach KD, Crawford JT, Bates JH: Repetitive Sequences as Probes for Mycobacterium tuberculosis. J Clin Mlcrobiol 1988, 26:2240-2245.

43. van Soolingen D, Qian L, de Haas PE, Douglas JT, Traore H, Portaels F, Qing HZ, Enkhsaikan D, Nymadawa P, van Embden JD: Predominance of a single genotype of Mycobacterium tuberculosis in countries of east Asia. J Clin Microbiol 1995, 33(12):3234-3238.

44. Sebban M, Mokrousov I, Rastogi N, Sola C: A data-mining approach to spacer oligonucleotide typing of Mycobacterium tuberculosis. Bioinformatics 2002, 18(2):235-243.

45. Dos Vultos T, Mestre O, Rauzier J, Golec M, Rastogi N, Rasolofo V, Tonjum T, Sola C, Matic I, Gicquel B: Evolution and Diversity of Clonal Bacteria: The Paradigm of Mycobacterium tuberculosis. PLOS ONE 2008, 3(2):e1538.

46. Warren RM, Streicher EM, Sampson SL, Van Der Spuy GD, Richardson M, Nguyen D, Behr MA, Victor TC, Van Helden PD: Microevolution of the Direct Repeat Region of Mycobacterium tuberculosis: Implications for Interpretation of Spoligotyping Data. J Clin Microbiol 2002, 40:4457-4465.

47. Nahid P, Bliven EE, Kim EY, Mac Kenzie WR, Stout JE, Diem L, Johnson JL, Gagneux S, Hopewell PC, Kato-Maeda M: Influence of M. tuberculosis Lineage Variability within a Clinical Trial for Pulmonary Tuberculosis. Plos One 2010, 5(5):e10753.

48. Sola C, Ferdinand S, Mammina C, Nastasi A, Rastogi N: Genetic diversity of Mycobacterium tuberculosis in Sicily based on spoligotyping and variable number of tandem DNA repeats and comparison with a spoligotyping database for population-based analysis. J Clin Microbiol 2001, 39(4):1559-1565.

49. Cheng SJ, Thibert L, Sanchez T, Heifets L, Zhang Y: pncA mutations as a major mechanism of pyrazinamide resistance in Mycobacterium tuberculosis: spread of a monoresistant strain in Quebec, Canada. Antimicrob Agents Chemother 2000, 44(3):528-532.

50. Niemann S, Kubica T, Bange FC, Adjei O, Browne EN, Chinbuah MA, Diel R, Gyapong J, Horstmann RD, Joloba ML, et al: The Species Mycobacterium africanum in the Light of New Molecular Markers. J Clin Microbiol 2004, 42(9):3958-3962.

51. R: A Language and Environment for Statistical Computing. [http://cran.rproject.org/].

52. Mezard M, Montanari A: Information, Physics, and Computation. Oxford University Press; 2009.

\section{doi:10.1186/1471-2105-12-224}

Cite this article as: Borile et al:: Using affinity propagation for identifying subspecies among clonal organisms: lessons from $M$. tuberculosis. BMC Bioinformatics 2011 12:224. 